\title{
Envelhecimento da População Brasileira: Reflexões e Aspectos a Considerar Quando da Definição de Desenhos de Pesquisas para Estudos Populacionais
}

\author{
RENATO P. VERAS* \\ SIDNEY DUTRA**
}

\section{Características demográficas do envelhecimento}

Ao contrário da crença geral, a principal característica demográfica do processo de envelhecimento de uma população não é o prolongamento do período de vida de seus indivíduos em decorrência da redução da mortalidade, mas a diminuição de sua fertilidade.

O número de pessoas de sessenta anos e mais é amplamente determinado pelo número de nascimentos sessenta a oitenta anos antes da database e a taxa de sobrevivência do nascimento até a velhice. $O$ número de pessoas idosas aumentou rapidamente em todo o mundo nos últimos cem anos, por causa de aumentos tanto no número de nascimentos quanto, na maioria dos países, nas taxas de sobrevivência.

É importante reconhecer que nossa futura população idosa, pelo menos a de daqui a sessenta anos, já nasceu. É possível traçar o impacto futuro

* Professor Adjunto do Instituto de Medicina Social da UERJ.

** Pesquisador e Mestrando do Instituto de Medicina Social da UERJ. 
que essa coorte de nascimentos terá sobre o tamanho dessa população mais velha bem antes de ela atingir a idade de sessenta anos. Por exemplo, as grandes coortes de nascimento de 1945-1960, o boom de nascimentos ocorrido no após-guerra na maioria dos países desenvolvidos, criarão, sob condiçōes de sobrevivência favoráveis, maior expansão do número de pessoas idosas no começo do próximo século. Se as condições sociais continuarem a melhorar no mundo, diminuindo a mortalidade precoce, a população idosa aumentará ainda mais do que o número de nascimentos.

Em comparação com o decréscimo da fertilidade, quedas nas taxas de mortalidade tendem a contribuir relativamente pouco para o aumento na proporção de pessoas idosas. Na verdade, podem contribuir para um decréscimo nessa proporção. Quedas nas taxas de mortalidade idade-específicas podem produzir diferentes efeitos sobre a estrutura etária, pois tudo depende das idades em que ocorrem. Se a queda é a mesma para todas as idades, a estrutura etária da população tende a permanecer a mesma; se as taxas de mortalidade são altas e o decréscimo na mortalidade é principalmente no grupo mais jovem, a proporção entre criạnças e jovens tenderá a aumentar, e a proporção de pessoas idosas tenderá a diminuir. Se, no entanto, a taxa de mortalidade cai no grupo idoso, como se espera que ocorra futuramente em países com baixa mortalidade, a proporção de pessoas idosas tenderá a aumentar.

\section{A transição epidemiológica}

Um índice de mortalidade conveniente, embora resumido, usado com freqüência para comparações não só entre países mas internamente também, é a expectativa de vida.

Em 1870, na Inglaterra, a expectativa de vida era de apenas 26 anos e quase metade da população tinha menos de 20 anos. ${ }^{1}$ Nos Estados Unidos, desde a virada do século e até 1980 , a expectativa de vida ao nascer aumentou de 47,0 para 73,6 anos. ${ }^{2}$ No Brasil, as mudanças são ainda mais impressionantes, já que a expectativa de vida dos brasileiros dobrou ao longo do século, como se pode ver na tabela 1.

1. MIDWINTER E., "An ageing world: the equivocal response". Ageing and Society, 10:221-228, 1990

2. OLSHANSKY S. J., e AULT B., "The fourth stage of the epidemiologic transition: the age of delayed degenerative diseases". Milbank Memorial Fund Quarterly/Health and Society, 64(3):355-391, 1986. 
TABELA 1

Expectativa de vida ao nascer para ambos os sexos, Brasil, 1900-2025

\begin{tabular}{ll}
\hline Anos & Expectativa de vida ao nascer \\
\hline 1900 & 33.7 \\
1910 & 34.1 \\
1920 & 34.5 \\
1930 & 36.5 \\
1940 & 38.5 \\
1950 & 43.2 \\
1960 & 55.9 \\
1970 & 57.1 \\
1980 & 63.5 \\
2000 & 68.6 \\
2020 & 72.1 \\
2025 & 75.3 \\
\end{tabular}

A expectativa de vida aumentou rapidamente neste século porque as doenças infecciosas e parasitárias cederam lugar às doenças cardíacas e ao câncer. Essa mudança nos padrōes de doença foi descrita por Omran ${ }^{3}$ como uma transição epidemiológica. De acordo com essa teoria, as nações, ao se modernizar, tendem a melhorar suas condições sociais, econômicas e de saúde. As condições de vida que anteriormente levavam à expansão de doenças infecciosas e parasitárias são rapidamente substituídas por melhores condições sanitárias e por uma tecnologia médica mais desenvolvida. Como o risco de morte por doenças infecciosas se reduz, os que escaparam de morrer dessas doenças sobrevivem até a meia-idade ou mais, enfrentando nesta faixa um risco aumentado de morte por doenças degenerativas ou causadas pelo homem. Em idades avançadas, as doenças degenerativas tendem a matar mais que as infecciosas. Por isso, essa

3. OMRAN A. R., "The epidemiologic transition: a theory of epidemiology of population change". Milbank Memorial Fund Quarterly, 49(4):509-538, 1971. 
transição nas causas de morte é geralmente caracterizada por uma redistribuição de mortes do jovem ao idoso. Conceitualmente, a teoria da transição epidemiológica se concentra nas complexas mudanças nos padrōes de saúde e doença e nas interações desses padrões com seus determinantes e suas consequêencias demográficas, econômicas e sociológicas. A teoria da transição epidemiológica descreve três estágios, que representam uma mudança que parte de mortalidade alta/fertilidade alta e chega em mortalidade baixa/fertilidade baixa. Omran distingue três estágios principais da transição epidemiológica.

A "idade da pestilência e da fome", quando a mortalidade é alta e flutuante, impedindo assim o crescimento populacional sistemático. Neste estágio, a expectativa de vida ao nascer é baixa e variável, situando-se entre vinte e quarenta anos.

A "idade da pandemia retrocessa", quando a mortalidade declina progressivamente. A taxa desse declínio se acelera à medida que os picos epidêmicos se tornam menos freqüentes ou desaparecem. Durante este estágio, a expectativa de vida ao nascer aumenta para cerca de cinqüenta anos. O aumento populacional é constante e começa a descrever uma curva exponencial.

A "idade da doença degenerativa e de doenças causadas pelo homem", quando a mortalidade continua a cair e eventualmente alcança a estabilidade num nível relativamente baixo. O efeito da longevidade é uma expectativa de vida que alcança os setenta anos e futuramente mudará pouco.

Quando essa teoria foi publicada, acreditava-se, de um modo geral, que o limite biológico da vida era atingido na septuagésima década. Agora chegou-se a um novo estágio, caracterizado por um rápido declínio nas taxas de mortalidade causada pelas doenças degenerativas principais. ${ }^{4} \mathrm{O}$ quarto estágio foi assim descrito:

A "idade da doença degenerativa adiada", quando a maioria das mortes se dá em idade avançada e praticamente no mesmo ritmo para homens e mulheres. Em relação ao terceiro estágio, a idade-padrão de mortalidade por morte natural continua a mesma, mas a distribuiçāo etária de mortes por causas degenerativas muda progressivamente para idades mais avançadas. Outra característica é a sobrevivência de mais pessoas com idades mais avançadas.

Existe considerável conjectura e controvérsia em relação aos padrōes futuros de morbidade. Fries ${ }^{5,6}$ argumenta que existem freios biológicos na

4. OLSHANSKY S. J., e AULT B., op.cit. 
vida humana e prognostica um declínio continuado em mortes prematuras, bem como a emergência de um padrão de morte natural ao fim de um espectro natural de vida. Por sua vez, Gruenberg ${ }^{7}$ e Kramer ${ }^{8}$ acreditam que, à medida que a expectativa de vida aumenta, haverá um aumento drástico e catastrófico na prevalência de distúrbios mentais e doenças crônicas em idosos. Thomas ${ }^{9}$ defende um ponto de vista mais otimista e prevê que a maioria das doenças da humanidade será controlada, podendo-se antever a possibilidade de uma sociedade humana relativamente livre de doenças. A opinião de Manton ${ }^{10}$ é de que a severidade das doenças crônicas será gradualmente reduzida, resultando em taxas menores de mortalidade e num aumento da expectativa de vida.

Como resultado desse novo quarto estágio da transição epidemiológica, a geração mais velha dos países desenvolvidos viu declinar notavelmente a mortalidade e experimentar ganhos na expectativa de vida em idades avançadas. ${ }^{11,12}$ Nos Estados Unidos, a taxa de mortalidade do grupo de oitenta anos ou mais está declinando mais rapidamente que a de qualquer outro grupo idoso. Seu número absoluto e sua percentagem na população estão crescendo numa taxa estarrecedora. Espera-se que, por volta do ano 2040, 12.834 .000 cidadãos norte-americanos terão 85 ou mais anos..$^{13,14}$

Quando um país tem um grande número de pessoas idosas, devem-se fazer esforços no sentido de se distinguir entre o terceiro e o quarto estágio da transição epidemiológica. A situação do Brasil, bem como a de muitos outros países, é bem diferente da dos países desenvolvidos, porque ainda

5. FRIES J. F., “Aging, natural death and the compression of mortality", N. Engl. J. Med., 303:130-135, 1980.

6. FRIES J. F., e CRAPO L. M., Vitality and Aging: Implications of the Rectangular Curve. WH Freeman and Company, San Francisco, 1981.

7. GRUENBERGE. M., The failures of success. Milbank Memorial Fund Quarterly/Health and Society, 55(1):3-24, 1977.

8. KRAMER M., The Rising Pandemic of mental disorders and associated chronic diseases disorders. Volume Acta Psychiatry Scandinavica 62 of Supplement 285, Epidemiological Research as basis for the Organization of Extramural Psychiatry, 1980.

9. THOMAS L., Biomedical Science and Human Health: the Long-Range Prospect. Issued as Proceedings of American Academy of Art and Sciences 106(3), 1977.

10. MANTON K. G., "Changing concepts of morbidity and mortality in the elderly population". Milbank Memorial Fund Quarterly, 60(2):183-244, 1982.

11. LOPEZ A. D., e HANADA K., "Mortality patterns and trends among the elderly in developed countries". Wld Hlth statist. Quart., 35(3/4):203-224, 1982.

12. MANTON K.G., "Mortality patterns in developed countries". Comparative Social Research, 7:259$286,1984$.

13. JETTE A. M., e M.B.J. "The graying of america, opportunities for physical therapy". Phys Ther, 67(10):1537-42, 1987 Oct.

14. ROSENWAIKE I., "A demographic portrait of the oldest old". Milbank Mem Fund Q, 63:187-205, 1985. 
nāo está à vista o quarto estágio de envelhecimento. Em conseqüência, por algumas décadas, o Brasil será um país com grande número de jovens e de idosos jovens. ${ }^{15,16}$

Apesar do grupo etário dos idosos se constituir de idosos mais novos, este fato não pode ser usado para minimizar a importante proporção de idosos no Brasil. Por exemplo, as projeçōes para um período de 75 anos, compreendido entre os anos de 1950 e 2025, indicam um crescimento de $1.514,3 \%$ da população idosa. Esta é a maior taxa de crescimento entre os países com populaçāo idosa acima de 15 milhões em 2025. ${ }^{17}$ Fato importante a observar é que nesse período a populaçāo brasileira, como um todo, estará aumentando cinco vezes, enquanto o grupo etário de sessenta anos ou mais estará se tornando quinze vezes maior.

\section{Apoio à pesquisa}

Apesar de atualmente já possuir uma população idosa numerosa, a produção acadêmica sobre o tema ainda é muito pequena no Brasil. Essa escassez talvez reflita a nossa situação econômica. Em um país que enfrenta crise econômica há mais de uma década, a alocação de recursos para pesquisa é menor do que a necessária. No entanto, tão grave quanto a falta de apoio financeiro e/ou políticas de investimento na área da ciência e tecnologia são algumas opiniōes, bastante equivocadas, que defendem a tese de que o investimento para a pesquisa na área da terceira idade pode ser aplicado em outros campos, visto que a produção internacional, particularmente americana e inglesa, é bastante extensa e suficiente para o delineamento global da situação dos idosos e para a tomada de decisōes.

Deve-se combater esta visão. O crescimento demográfico brasileiro tem características particulares que precisam ser apreendidas através de estudos e desenhos de investigação que dêem conta dessa especificidade.

15. GRIGSBY J. S., "Paths for future population aging". The Gerontologist, 31(2):195-203,1991.

16. VERAS R. P., "Avaliação multidimencional da população idosa". $39^{\circ}$ Reuniāo Anual da Sociedade Brasileira para o Progresso da Ciência (SBPC), 39(7):90, 1987.

17. Veja maiores informaçōes em Kalache [KALACHE A., VERAS R. P. e RAMOS L.R., "O envelhecimento da populaçāo mundial; um desafio novo". Revista de Saúde Pública, São Paulo, USP, 21(3):200-10, 1987; KALACHE A., "Ageing in developing countries". In Principles and Practice of Geriatric Medicine, p. 1517-1528, John Wiley \& Sons Ltd, 1991.] e Veras [VERAS R. P., A survey of the health of elderly people in Rio de Janeiro, Brazil. $\mathrm{PhD}$ thesis, Division of Psychiatry, United medical \& Dental Schools, Guy's Hospital, University of London, London, 1992; VERAS R. P., 1987, op. cit.; VERAS R. P., "Consideraçoes acerca de um jovem país que envelhece". Cadernos de Saúde Pública, 4(4):382-397, 1988; VERAS R. P., "Brazil is getting older: demographic changes and epidemiological challenges". Revista de Saúde Pública, 25(6) 1991] e Warnes [WARNES T., "The elderly in less-developed world regions". Ageing and Society, 6:373-380,1986. 
A pesquisa corretamente orientada deve se transformar, por sua vez, em subsídio para a implantação de programas adequados à realidade do país.

\section{Brasil, país heterogêneo}

Indicadores que têm como denominador o país como um todo apresentam informações bastante enganosas em países com as características do Brasil. Nosso país apresenta uma conjunção de muitos contrastes e profundas desigualdades que requerem consideração em um estudo rigoroso. $O$ Brasil possui regiões tão miseráveis quanto aquelas dos países mais pobres do mundo e, ao mesmo tempo, metrópoles onde segmentos sociais desfrutam de facilidades e serviços típicos dos países desenvolvidos. Apesar do segmento rico da população ser proporcionalmente pequeno, o Brasil tem uma das dez maiores economias do mundo. A distribuição da riqueza, porém, se caracteriza pela iniquidade e a concentração de recursos nas mãos de relativamente poucos. Mesmo assim, em valores absolutos, é elevado o número de pessoas com alto poder aquisitivo.

Os idosos brasileiros conseguiram sobreviver a condiçōes adversas. Expressam, portanto, uma seleção social e biológica diferenciada. Ao se observar a distribuição espacial da população idosa percebe-se que esta se concentra nas grandes cidades e nos bairros com maiores facilidades, onde vive a população de maior poder aquisitivo. Isto não quer dizer que os idosos sejam uma parcela rica da população, mas os que sobreviveram até idades mais avançadas são, em média, menos pobres que o conjunto da sociedade. É, no entanto, importante frisar que este quadro atual tende a mudar. Em paises de Terceiro Mundo, onde quase não existem serviços e programas sociais e de saúde para o idoso, aqueles de baixo poder aquisitivo tendem a ter uma qualidade de vida pior, morrendo mais cedo. Ramos, em estudo realizado em São Paulo, utilizou como indicador socioeconômico o número de idosos residentes nos distritos, e os resultados demonstraram ser consideravelmente maior a proporção de idosos nas regiões mais ricas da cidade. Mesma metodologia foi aplicada e se mostrou correta em estudo no Rio de Janeiro. ${ }^{18}$

Portanto, tratar um país como o Brasil, nos desenhos de projetos de pesquisa médico-sociais, pela média - isto é, considerar uniforme sua estrutura social, econômica e demográfica - é um erro metodológico e de graves conseqüências para o planejamento, por distorcer a realidade, impedindo sua correta apreensão. Desenhos que não considerem a hetero-

18. VERAS R. P, A survey of the health of elderly people in Rio de Janeiro, Brazil, op.cit, 1992. 
geneidade de países, ou de grandes metrópoles, nāo conseguem captar corretamente as reais necessidades e demandas dos diferentes grupos sociais.

\section{País jovem de cabelos brancos}

Outros aspectos também devem ser considerados quando se pensa no processo de envelhecimento da população idosa brasileira.

Apesar do aumento da população de idosos, o Brasil possui, e ainda possuirá por algum tempo, uma vasta população jovem..$^{19}$ Esta situação difere em muito dos países do hemisfério norte, onde o número de jovens é pequeno, estável ou mesmo decrescente há algumas décadas, e a população idosa, sobretudo em suas faixas mais avançadas, continua a crescer. Conforme assinalado, o crescimento populacional brasileiro tem características distintas das dos países centrais. Aqui, apesar do grupo etário jovem tender a reduzir-se proporcionalmente, este grupo ainda é expressivo. Agora estamos diante de um importante crescimento do grupo etário idoso. Estes fatos colocam o Brasil frente a um novo e grande desafio: a alocação de recursos para atender às necessidades das faixas etárias jovem e idosa, ambas carentes de recursos e de grande demanda de serviços. Assim, os recursos para os programas sociais deverão ser destinados não apenas para combater as altas taxas de mortalidade infantil e a desnutrição, ou prover os programas educacionais, mas também para combater as doenças crônicas como artrite, cardiopatias, doenças degenerativas, debilidades cognitivas, entre outros males típicos da velhice. Acrescente-se aos problemas de saúde os benefícios e pensões da seguridade social a que todos os idosos têm direito. Em termos econômicos coloca-se um grande desafio: dois grupos economicamente não produtivos demandam os parcos recursos de um país carente e de pequena tradiçāo de investimento na área médico-social.

\section{Mulher idosa}

O problema da mulher idosa merece ser enfatizado e reconhecido em sua especificidade, recebendo maior ênfase entre os pesquisadores e estudiosos. Vários indicadores atestam a importância de maior atenção a esta área. A situação conjugal da população idosa do país mostra que $68 \%$ das pessoas desácompanhadas (solteiras, viúvas e descasadas) são do sexo

19. Para efeito deste estudo, considera-se 0 a 14 anos populaçāo jovem e 60 anos ou mais, populaçāo idosa. 
feminino. ${ }^{20,21}$ Por força de fatores culturais, a mulher se casa mais cedo do que o homem. Como sua expectativa de vida é maior, ela se acha mais sujeita à solidão na velhice - casando-se mais cedo e vivendo mais. $O$ panorama fica ainda mais cinza diante da constatação de que, na maioria das vezes, a solidão vem acompanhada de pobreza e de doenças crônicas. O tripé solidão-pobreza-doença atinge boa parte das mulheres no final da vida. $22,23,24,25$ Estas características se mostram presentes na maioria das idosas brasileiras, aliás de modo semelhante àquelas dos países do Primeiro Mundo. $26,27,28$

Os programas dirigidos à mulher precisam incorporar aquelas de mais de sessenta anos. A esta maioria idosa a sociedade deve tributos, posto que, como mulheres, desempenharam múltiplos papéis, como trabalhadoras na reprodução da vida material ou como mães, reproduzindo a vida biológica da sociedade. Os problemas médicos sociais dos idosos são eminentemente os da mulher.

\section{Diferenças regionais}

O último censo brasileiro ${ }^{29}$ apresentou os estados da Para íba e do Rio de Janeiro como aqueles que abrigam a maior proporção da população idosa do país (em torno de $9 \%$ ). No entanto, estes números devem ser analisados. Na Paraíba o processo de envelhecimento decorreu principalmente das péssimas condições de vida de sua população produtiva, que migra em grande proporção para outros estados em busca de trabalho. Assim, o crescimento proporcional da população idosa está ligado ao processo de

20. BERQUÓ E. S., e MOTTA LEITE V., "Algumas consideracões sobre a demografia da populaçāo idosa no Brasil". Ciência e Cultura, Sāo Paulo, 40(7):679-688, 1988.

21. BERQUO E. S., e CAZENAGHI S.M., "Oportunidades e fatalidades: um estudo demográfico das pessoas que moram sozinha". Anais do VI Encontro Nacional de Estudos populacionais, 1:155-181, 1988.

22. CREECY R.F., BERG W.E., e R. WRIGHT J., "Loneliness among the elderly: a causal approach". Journal of Gerontology, 40(4):487-493, 1985.

23. JONES D.A., VICTOR C.R. e VETTER N.J., "The problem of loneliness in the elderly in the community: characteristics of those who are lonely and the factors related to lonelines".Journal of the Royal College of General Practitioners, 35:136-139, 1985.

24. WARLICK J. L., "Why is poverty after 65 a woman's problem". Journal of Gerontology, 40(6): 751$757,1985$.

25. PADGETT D., "Aging minority women: issues in research and health policy". Women Health, 14(3-4):213-25, 1988.

26. CREECY R.F., BERG W.E. e R. WRIGHT J., op. cit., 1985.

27. JONES D.A., VICTOR C.R. E VETTER, N.J., op. cit., 1985.

28. BERQUÓ E.S., e MOTTA LEITE V., op. cit., 1988.

29. Resultados preliminares do censo de 1991. 
migração, aliado às ainda altas taxas de mortalidade da população jovem e à drástica redução da fecundidade, por causa de programas de esterilização em massa nas regiões carentes. Apesar de estar localizada em uma região onde os laços sociais são bastante fraternos e a ajuda mútua é um traço cultural bastante presente, a miséria, a pobreza e a migração tornam irreais, destinados ao fracasso, programas concebidos no apoio social e/ou familiar.

No Rio de Janeiro a situação é diferente. Sua população é mais estável, e o processo de redução de fecundidade e de mortalidade já ocorre há vários anos. Embora o envelhecimento de sua população se dê em bases mais próximas dos padrões europeus e de outros países do Primeiro Mundo, também tem suas particularidades que precisam ser apreendidas, para uma melhor e mais eficiente definição de prioridades. Trata-se, portanto, de realidades distintas em duas regiōes brasileiras, apesar de ambas levarem ao envelhecimento da população. Os estudos precisam apreender estas diferenças.

\section{Heterogeneidade das metrópoles brasileiras}

Assim como o Brasil, a cidade do Rio de Janeiro também apresenta grandes disparidades econômicas e sociais. As iniquidades da má distribuição de renda se refletem na distribuição geográfica da população pelo território do município. Existem áreas com boa estrutura de serviços, que concentram os estratos mais ricos. Por outro lado, há locais que abrigam uma enorme concentração de miséria quase absoluta, carentes de serviços essenciais, como saneamento básico, transporte, assistência médica, educação, entre outros.

Apesar deste convívio de condições tão precárias e insuficientes ao lado de condições fartas ser uma característica das grandes metrópoles do Brasil e do Terceiro Mundo, não se deve olhar a cidade como um todo homogêneo, mas sim assumir as diferenças socioeconômicas nela presentes, a fim de que o estudo propicie uma visão mais correta da realidade e dê bases mais fidedignas para o planejamento. O mais adequado, para nós, é examinar separadamente cada região da cidade, considerando suas realidades especificas, a fim de se obter um resultado que informe e reflita mais corretamente as condições de vida dos vários segmentos sociais. Deste modo, a cidade deve ser avaliada pelas 'áreas homogêneas', isto é, bairros e segmentos sociais com padrões similares de facilidades. ${ }^{30}$

30. RAMOS L. R., Health care for the elderly - an unmet need. Health and living conditions of the elderly 
Caso esta preocupação não seja levada em consideração, obtêm-se resultados de pouca eficácia no planejamento de saúde. Para reforçar e demonstrar a correção da abordagem apresentada - que leva em consideração as iniquidades socioeconômicas do país e/ou das grandes metrópoles, e que também observa as diferentes proporções de sexo e de faixas etárias dos idosos nos distritos e bairros das cidades brasileiras - apresentaremos nas páginas seguintes alguns resultados de um estudo recentemente concluído no Rio de Janeiro, ${ }^{31}$ que no seu desenho levou em consideração as observaçōes aqui assinaladas.

\section{Resultados e análises}

O estudo ${ }^{32}$ teve a preocupação de selecionar três distritos da cidade do Rio de Janeiro, representantes de segmentos populacionais de diferentes estruturas socioeconômicas.

Foi realizado um inquérito domiciliar em três áreas do Rio de Janeiro - Copacabana, Méier e Santa Cruz - utilizando como fonte para a coleta das informações o questionário multidimensional Boas, que cobre vários aspectos da vida dos idosos. ${ }^{33,34,35,36,37,38,39}$

population living in a large urban center in Brazil - Sāo Paulo. Department of Community Health, London School of Hygiene and Tropical Medicine (LSHTM) London, Project Report, November, 1985.

31. VERAS R.P., A survey of the health of elderly people in Rio de Janeiro, Brazil, op. cit., 1992.

32. VERAS R.P., A survey of the health of elderly people in Rio de Janeiro, Brazil, op. cit., 1992.

33. BOAS (Brazil Old Age Schedule) Questionnaire - Multidimensional questionnaire for the elderly population. Instituto de Medicina Social, UERJ (University of the State of Rio de Janeiro), 1987. Rio de Janeiro.

34. BOAS (Brazil Ode Age Schedule) Manual. Instituto de Medicina Social, UERJ, 1987. Rio de Janeiro.

35. VERAS R.P., SOUZA C.A.M., CARDOSO R.F., MILIOLI R., e DUTRA S., "Pesquisando populações idosas - a importância do instrumento e o treinamento de equipe: uma contribuiçāo metodológica”. Revista Saúde Pública, São Paulo, 22(6):513-8, 1988.

36. VERAS R.P., COUTINHO E. e GEORGE N. Jr., "População idosa no Rio de Janeiro (Brasil): estudo-piloto da confiabilidade e validação do segmento de saúde mental do questionário BOAS". Revista de Saúde Pública. Sāo Paulo, USP, 24(2):156-63, 1990.

37. VERAS R.P., e COUTINHO E., "Estudo de prevalência de depressāo e síndrome cerebral orgânica na populaçāo de idosos em três distritos da cidade do Rio de Janeiro. Revista de Saúde Pública, 25(3):209-217, 1991.

38. VERAS R.P., e MURPHY E., "The ageing of the third world: tackling the problems of community surveys - Part I: Brazil - a young country grows old". International Journal of Geriatric Psychiatry, 6(9):619-627, 1991.

39. VERAS R.P., e MURPHY E., "The ageing of the third world; tackling the problems of community surveys - Part II: Brazil - a young country growsold". InternationalJournal of Geriatric Psychiatry, 6(9):629-637, 1991. 
Este trabalho produziu um grande conjunto de informações. Aqui, a intenção é apresentar apenas alguns exemplos, que reafirmem a correção das preocupaçōes apresentadas, sem aprofundar a análise estatística dos dados.

Neste artigo vamos discutir alguns achados cobertos pelas seções de saúde física, utilização de serviços de saúde e atividades diárias do idoso.

Iniciamos a apresentação das informações produzidas pelo estudo com dados demográficos. Na tabela 2 observa-se a distribuição por nível de escolaridade e número de pessoas que residem com o idoso entrevistado. Uma característica do idoso é que o nível de escolaridade é baixo em todas as áreas, particularmente entre as mulheres, com ênfase nas regiões mais pobres da cidade. Viver só é predominante entre as mulheres em todos os distritos estudados, com maior número em Copacabana, região mais rica do estudo. Possivelmente associada à pobreza, a maior proporção de idosos residindo em uma mesma residência foi encontrada em Santa Cruz.

\section{TABELA 2}

Indicadores demográficos da amostra, por sexo e distritos: nível educacional e número de pessoas residindo no mesmo domicílio.

\begin{tabular}{|c|c|c|c|c|c|c|}
\hline \multirow[b]{2}{*}{ Indicadores $\left({ }^{*}\right)$} & \multicolumn{2}{|c|}{ Copacabana } & \multicolumn{2}{|c|}{ Méier } & \multicolumn{2}{|c|}{ S. Cruz } \\
\hline & Masculino & Feminino & Masculino & Feminino & Masculino & Feminino \\
\hline sem educação & 2.22 & 9.15 & 29.79 & 2.76 & 55.32 & 68.53 \\
\hline primário & 8.89 & 14.38 & 25.53 & 28.97 & 22.34 & 20.98 \\
\hline secundário & 41.11 & 58.17 & 41.49 & 26.90 & 19.15 & 10.49 \\
\hline superior & 47.78 & 18.30 & 3.19 & 1.38 & 3.19 & 0.00 \\
\hline total & 100.00 & 100.00 & 100.00 & 100.00 & 100.00 & 100.00 \\
\hline total* & $90.00^{(a)}$ & $153.00^{(\mathrm{b})}$ & $94.00^{(c)}$ & $145.00^{(\mathrm{d})}$ & $94.00^{(\mathrm{e})}$ & $143.00^{(f)}$ \\
\hline mora só & 11.96 & 18.13 & 3.13 & 8.78 & 9.38 & 14.38 \\
\hline 1 ou 2 & 71.74 & 58.75 & 63.54 & 56.08 & 38.52 & 32.19 \\
\hline 3 ou 4 & 10.87 & 19.38 & 20.83 & 23.65 & 25.00 & 24.66 \\
\hline 5 ou mais & 5.43 & 3.75 & 12.50 & 11.49 & 27.10 & 28.77 \\
\hline total & 100.00 & 100.00 & 100.00 & 100.00 & 100.00 & 100.00 \\
\hline total* & 92.00 & 160.00 & 96.00 & 148.00 & 96.00 & 146.00 \\
\hline
\end{tabular}


Na tabela 3, a amostra também confirmou os dados internacionais: ${ }^{40} \mathrm{O}$ número de pessoas casadas após os sessenta anos é superior no sexo masculino e os casos de viuvez são muito mais numerosos entre as mulheres.

TABELA 3

Indicadores demográficos da amostra, por sexo e distrito: situação conjugal.

\begin{tabular}{|c|c|c|c|}
\hline Categoria & Masculino (\%) & 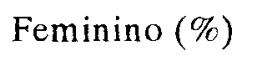 & total $(\%)$ \\
\hline & \multicolumn{2}{|c|}{ Copacabana } & \\
\hline casado & $69(58.97)$ & $48(41.03)$ & $117(100)$ \\
\hline viúvo & $12(13.04)$ & $80(86.96)$ & $92(100)$ \\
\hline \multirow[t]{2}{*}{ outros } & $11(25.58)$ & $32^{-}(74.42)$ & $43(100)$ \\
\hline & \multicolumn{2}{|c|}{ Méier } & \\
\hline casado & $82(57.34)$ & $61(42.66)$ & $143(100)$ \\
\hline viúvo & $12(14.81)$ & $69(85.19)$ & $81(100)$ \\
\hline \multirow[t]{2}{*}{ outros } & $2(10.00)$ & $18(90.00)$ & $20(100)$ \\
\hline & \multicolumn{2}{|c|}{ S. Cruz } & \\
\hline casado & $67(57.76)$ & $49(42.24)$ & $116(100)$ \\
\hline viúvo & $20(20.62)$ & $77(79.38)$ & $97(100)$ \\
\hline outros & $9(31.03)$ & $20(68.97)$ & $29(100)$ \\
\hline
\end{tabular}

Nota: A categoria "casado" inclui todos aqueles que estāo vivendo juntos (casado, amigado etc). A categoria "outros" inclui todas as demais possibilidades, isto é, solteiros, divorciados, separados.

40. MYERS G. C., e NATHANSON C. A., Ageing and the family. World Health Statistic, World Health Organization, 35(3/4):225-238, 1982. 
A proporção de entrevistados que reportaram problemas de saúde foi superior entre as mulheres, valendo ressaltar que a imensa maioria $(82,5 \%)$ do grupo de idosos entrevistados nāo relata problemas físicos ou queixas de saúde. Esta informação é coerente com outros estudos comunitários. ${ }^{41,42}$ No entanto, entre os que reportaram problemas de saúde, $64.4 \%$ responderam possuir mais de uma queixa. Esta é uma característica importante nos idosos, que deve ser levada em consideração quando da organização dos serviços de saúde. Acresce-se a este fato que os problemas de saúde de idosos, além de serem de longa duração, requerem pessoal qualificado, equipe multidisciplinar, equipamentos e exames complementares de alto custo. Apesar de uma importante parcela deste grupo etário estar bem de saúde, entre aqueles que utilizaram serviços de saúde nos três meses anteriores à entrevista, $59.0 \%$ retornaram uma ou mais vezes para novas consultas. As mulheres usaram os serviços de saúde com mais freqüência do que os homens nas três áreas estudadas. A maior proporção de consultas foi em Copacabana e a menor em Santa Cruz. No período de três meses anterior à entrevista, $4.88 \%$ dos entrevistados estiveram hospitalizados, sendo $3.25 \%$ na rede publica e $1.63 \%$ na rede privada. Como esperado, a imensa maioria dos idosos internados na rede publica é composta de residentes da região mais pobre da cidade. Esta taxa de internação é muito elevada para um período de apenas três meses, mesmo se tratando de uma população idosa. Pode-se especular que a falta de serviços domiciliares e/ou ambulatoriais faz com que o primeiro atendimento se realize no hospital. Outro aspecto a observar é que apesar de aproximadamente $20 \%$ dos idosos da amostra relatarem problemas de saúde, $80.26 \%$ usam regularmente remédios prescritos por médicos. Esse consumo é maior entre as mulheres nos três distritos estudados. O uso de medicamento após os setenta anos aumentou consideravelmente, fato também observado nos três distritos e em ambos os sexos. Em relaçāo à pergunta sobre o uso regular de medicamentos nāo prescritos por médicos, $27.2 \%$ idosos responderam afirmativamente. O maior consumo de medicamentos não prescritos ocorre entre o grupo de idosos jovens (60 a 69 anos) e entre os moradores de Santa Cruz. Uma hipótese a ser explorada para este maior consumo na região mais pobre pode ser a dificuldade ou a falta de serviços de saúde e de médico para prescrever o medicamento para a população dessa área, o que cria condiçōes para a sobrevivência de uma cultura

41. Pan American Health Organization (PAHO). A profile of the elderly in Guyana. 1989. Tecnical Paper No. 24, Washington.

42. LINDESAY J., "The Guy's/Age concern survey: Physical health and psychiatric disorder in an urban elderly community". International Journal of Geriatric Psychiatry, 5(3):171-178, 1990. 
informal de trocas de receitas entre a população, com alto risco de danos à saúde.

Considerando-se apenas os problemas de saúde mais prevalentes entre os idosos, existe uma frequência maior nas regiões mais pobres e carentes da cidade. Este fato, apesar de bastante conhecido para a população em geral, mostra a necessidade de não se excluírem os idosos quando a saúde for prioridade, dedicando a eles a atenção necessária dos órgãos do setor público.

Além dos problemas de saúde - que aparecem entre os máis apontados -, quando os entrevistados foram indagados sobre a principal carência e/ou dificuldade de seu cotidiano, o principal problema é o econômico. A falta de transporte e diversos problemas sociais também fazem parte do rol de queixas, conforme pode ser observado na figura 1. Este fato reforça a necessidade de que o cuidado com o idoso se dê de forma integral e não contemple apenas aspectos relacionados com a assistência à saúde.

\section{FIGURA 1}

Percepção dos idosos sobre as necessidades básicas não satisfeitas.

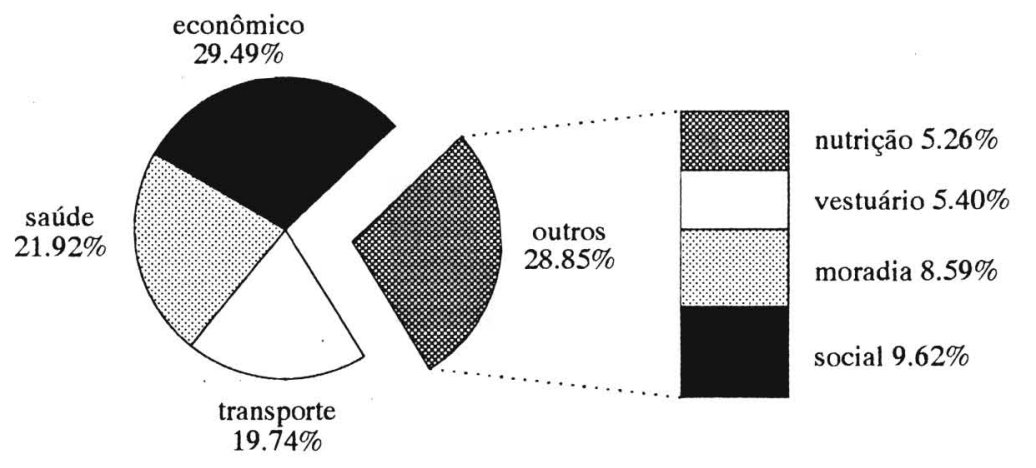

Um exemplo da necessidade de se associar indicadores socioeconômicos para apreender corretamente o perfil de saúde da população idosa pode ser observado na queixa relativa a dificuldade visual. Embora a maior proporção desta queixa se localize na região de Santa Cruz, o número de idosos com problemas de visão e que usam óculos é maior em Copacabana.

Outra área explorada no estudo refere-se às atividades do dia-a-dia do idoso. É sabido que o cuidado de saúde para com o idoso é bastante caro. Neste particular, a pesquisa epidemiológica pode propiciar os instrumentos mais adequados para maior eficiência na adoção de prioridades e alocação de recursos. 
No estudo com essa população, o uso de indicadores de morbidade para a avaliação do estado de saúde, apesar de importante, não tem o mesmo valor preditivo que apresenta nos outros grupos etários. A partir dos sessenta ou setenta anos a maioria das pessoas possui uma ou mais doenças. Assim, a identificação de um problema de saúde tem um grau de importância distinto do que ocorre nos demais grupos etários. Nessa fase da vida, mais importante é identificar se a doença está impedindo o idoso de desempenhar, de forma autônoma e independente, sua rotina de vida. Por exemplo, mais importante do que constatar uma doença como a artrite é saber se este problema está impedindo a locomoção do idoso (perda da independência) ou se suas atividades só são possíveis com a ajuda de uma cadeira de rodas e de alguém para empurrá-la (perda da autonomia).

Por isso, nos questionários multidimensionais para idosos, a seção intitulada atividades diárias, ADL na literatura inglesa (activity daily living), é um indicador muito sensível na definição da qualidade de vida do idoso e de grande valor para o planejamento de saúde.

No questionário Boas, na seção atividades diárias, há uma pergunta constituída de quinze itens concernentes à capacidade de desempenho das atividades diárias. Na análise das respostas, os entrevistados foram divididos em dois grupos: os que informaram ser capazes e os que não têm condições de executar a tarefa. Dois itens tiveram que ser excluídos da análise por causa de fatores culturais. A pergunta se o idoso era capaz de preparar sua comida e arrumar sua casa não se aplica, no nosso meio, ao sexo masculino. A maioria daqueles que gozam de perfeita autonomia e independência disse que estas tarefas sempre foram desempenhadas por uma mulher. À exceção destas, as demais questões foram analisadas, e a partir do conjunto de suas freqüências estes itens foram ordenados em uma escala e divididos em quatro grupos; isto é, itens com frequências similares foram agrupados. Em nosso estudo, a escala das atividades, da de mais fácil desempenho para a mais difícil, é a seguinte:

Nível um de dificuldade: 1. alimentar-se sozinho, 2. pentear o cabelo, 3. deitar-se e levantar-se da cama, 4. vestir-se.

Nível dois de dificuldade: 5. tomar banho, 6. caminhar em superfície plana, 7. usar o banheiro em tempo, 8. tomar remédios.

Nível três de dificuldade: 9. sair para perto de casa, 10. subir escadas.

Nível quatro de dificuldade: 11. cortar as unhas dos pés, 12. sair para distâncias longas, 13. tomar ônibus. 
TABELA 4

Percentual de idosos, por sexo e grupo etário, que conseguem executar um conjunto de atividades diárias, considerando-se quatro níveis de dificuldades.

\begin{tabular}{lccc}
\hline & Homens & \\
\hline AD & $60-69$ & $70-79$ & 80 ou + \\
grupo & $\mathrm{N}=135$ & $\mathrm{~N}=111$ & $\mathrm{~N}=38$ \\
& & & \\
nível 1 & $0.74 \%$ & $0.00 \%$ & $2.63 \%$ \\
nível 1 e 2 & $3.70 \%$ & $1.80 \%$ & $2.63 \%$ \\
nível 1,2 e 3 & $3.70 \%$ & $9.01 \%$ & $13.16 \%$ \\
nível 1,2,3 e 4 & $91.85 \%$ & $89.19 \%$ & $81.58 \%$ \\
\hline & & & \\
& & & \\
\hline & & & \\
AD & & & \\
grupo & $\mathrm{N}=226$ & $\mathrm{~N}=166$ & $\mathrm{~N}=59$ \\
& & & \\
nível 1 & $0.44 \%$ & $2.41 \%$ & $6.78 \%$ \\
nível 1 e 2 & $1.33 \%$ & $3.61 \%$ & $11.86 \%$ \\
nível 1,2 e 3 & $7.96 \%$ & $17.47 \%$ & $20.34 \%$ \\
nível 1,2,3 e 4 & $90.27 \%$ & $76.51 \%$ & $61.02 \%$ \\
\end{tabular}

Nota: (a) Existe uma perda de informaçāo no nível 2;

e (b) existem duas perdas no nível 4.

$\mathrm{Na}$ Tabela 4 demonstra que apesar das dificuldades na realização de simples atividades do dia-a-dia, o desempenho dos idosos é mais do que satisfatório. Poucos são os que se restringem apenas ao nível 1 de dificuldade (alimentar-se sozinho, pentear o cabelo, deitar-se e levantar-se da cama e vestir-se), sendo que a imensa maioria, em ambos os sexos e toda faixa etária, conseguem atingir itens mais difíceis da escala das atividades diárias.

Como descrito nos demais trabalhos, este estudo confirmou os achados anteriores: os mais idosos e as mulheres são os que apresentam pior 
desempenho. Os resultados ${ }^{43}$ mostraram que $30.9 \%$ dos entrevistados (sendo $18.42 \%$ no sexo masculino e $38.98 \%$ no sexo feminino) do grupo etário mais idoso reportaram problema no desempenho das atividades consideradas mais difíceis (nível 4), enquanto nos idosos jovens (60 a 69 anos) a proporção para igual dificuldade cai para $9,1 \%$ (sendo $8.15 \%$ no sexo masculino e $9.73 \%$ no feminino). Para desenvolver as atividades mais simples, entre aqueles com mais de oitenta anos, $5.1 \%$ não conseguiram desempenhar tais tarefas $(2.63 \%$ dos homens e $6.78 \%$ das mulheres $)$, enquanto entre os idosos do grupo etário compreendido entre 60 e 69 anos este percentual cai para $0.5 \%$ ( $0.74 \%$ dos homens e $0.44 \%$ das mulheres). Apesar de pequeno, o grupo nível 1 é constituído de idosos totalmente dependentes e que precisam de constante ajuda para todas as atividades do dia-a-dia, até as mais simples.

Analisando por sexo, as mulheres informaram maiores dificuldades que os homens para o desempenho de suas atividades, e os residentes nas regiões mais pobres tiveram um pior desempenho.

O uso deste indicador, extremamente simples, além do perfil da autonomia da vida do idoso, traz informações de grande interesse para o cuidado integral da pessoa idosa. Por exemplo, um dos problemas apresentados neste estudo refere-se à dificuldade dos idosos no Rio de Janeiro para o uso do transporte coletivo (ônibus). Para eles esta é, na escala o item de maior dificuldade de desempenho, em contraste com os resultados dos estudos europeus ${ }^{44}$ nos quais o uso de ônibus não constitui problema (cortar as unhas dos pés e caminhar a longas distâncias são as atividades listadas como as mais difíceis). Também no estudo de Ramos, em São Paulo, ficou constatado que o uso do ônibus por parte dos idosos é uma das maiores dificuldades deste grupo populacional. ${ }^{45}$ Medidas precisam ser urgentemente implementadas nessa área.

43. A descrição detalhada da metodologia empregada e maiores informaçōes podem ser obtidas em outro trabalho de um dos autores deste artigo [VERAS R. P., Brazil is getting older: demographic changes and epidemiological challenges. Op. cit., 1991.

44. HEIKKINEN E., WATERS W.E., e BRZESINSKI Z.J.The elderly in eleven countries; a sociomedical survey. World Health Organization (WHO), Public Health in Europe, No. 21, Copenhagen, 1983.

45. RAMOS L. R., Growing old in Säo Paulo, Brazil: assessment of health status and social support of elderly people from different socioeconomic strata living in the community. $\mathrm{PhD}$ thesis, London School of Hygiene and Tropical Medicine (LSHTM), London, 1986. 


\section{Conclusão}

O propósito deste artigo foi despertar o interesse em estudos que forneçam subsídios para a implantação de serviços e cuidados para os idosos, e também estimular os pesquisadores a aperfeiçoar indicadores e elaborar projetos mais detalhados e completos.

A apresentação de alguns resultados e informaçōes visou descrever o enorme campo de investigação a ser explorado e também reforçar nossa tese de que os desenhos devem estar em sintonia com as característica e realidades socioeconômicas e demográficas, principalmente quando se realiza um estudo exploratório sobre uma população nunca antes estudada.

\section{RESUMO}

\section{Envelhecimento da população brasileira: reflexões e aspectos a considerar quando da definição de desenhos de pesquisas para estudos populacionais}

Este artigo aborda questōes demográficas do envelhecimento, discute o processo de transiçāo demográfica, as particularidades do Brasil em termos de suas diferenças regionais e mostra a importância de pesquisas que considerem estas diferenças tendo em vista sua importância nāo só a nível do país mas também no interior das grandes metrópoles brasileiras. Os autores defendem que os estudos epidemiológicos que tem a preocupação de fornecer informaçōes para o planejamento mais criterioso para a organização de serviços devem se preocupar com esses fatos. Neste artigo apresentam os resultados de uma pesquisa recentemente realizada no Rio de Janeiro que ao invés de trabalhar com a cidade como um todo selecionou, através de indicadores sociais, econômicos e demográficos áreas homogêneas e representativos de três estratos da população. A partir daí pode-se identificar problemas específicos e demandas distintas. Recorte também foi feito por faixa etária e sexo mostrando que a distribuição de doenças e problemas dos idosos se dāo de forma diferente. Ênfase foi dado à mulher idosa e as atividades diárias do idoso. 


\section{ABSTRACT \\ Brazilian population's aging: reflections and aspects to be considered in the definition of research outlines for populational studies.}

The article focuses on aging's demographic issues, discusses the process of demographic transition. Brazil's peculiarities in terms of its regional differences and shows the importance of researches which deal with these differences, considering its importance, not only at the country's level, but also in major Brazilian metropoles.

According to the authors, epidemiological studies designed to give information for a more detailed planning for services' organization must be concerned with such facts.

The article presents the results of a research, recently in Rio de Janeiro that, instead of analysing the city as a whole, selected, through social, economic and demographic indicators, homogeneous and representative areas of three populational strata. Then it was possible to identify specific problems and distinct demands.

Also, it was made a division, according to sex and age group, showing that the distribution of diseases and problems among elderly people happens differently. Emphasis was given to aged women and older people's daily activities.

\section{RESUME}

Le vieillissement de la population brésilienne: réflexions et suggestions pour une définition d'un programme de recherche démographique.

Ce texte analyse les caracteristiques démographiques du Brésil et spécialement le vieillissement de sa population. Cette analyse a comme but définir la demande de la population agée de la ville de Rio de Janeiro particulièrement la population féminine - face à l'organization des services de santé. 